\title{
SCALE FACTOR IN DOUBLE PARTON COLLISIONS AND PARTON DENSITIES IN TRANSVERSE SPACE
}

\author{
A. Del Fabbro and D.Treleani \\ Università di Trieste; Dipartimento di Fisica Teorica, Strada Costiera 11, Miramare-Grignano, \\ and INFN, Sezione di Trieste, I-34014 Trieste, Italy.
}

\begin{abstract}
The scale factor $\sigma_{e f f}$, which characterizes double parton collisions in high energy hadron interactions, is a direct manifestation of the distribution of the interacting partons in transverse space, in such a way that different distributions give rise to different values of $\sigma_{e f f}$ in different double parton collision processes. We work out the value of the scale factor in a few reactions of interest, in a correlated model of the multi-parton density of the proton recently proposed.
\end{abstract}

E-mail delfabbr@trieste.infn.it

E-mail daniel@trieste.infn.it 


\section{INTRODUCTION}

Double parton collisions are a new feature of high energy hadron interactions which becomes increasingly important at high energies. The number of partons that can undergo a hard collision is in fact a fast growing function of $s$ and, as a consequence, when the c.m. energy is large enough, the probability of having more than one hard partonic interaction in the same inelastic event becomes sizable. Double parton collisions, foreseen long ago by several authors [1], have been in fact observed recently by CDF [2]. The non-perturbative input to the double parton collisions are the double parton distributions, which are independent on the parton distributions usually considered in large $-p_{t}$ physics, since they are related directly to the two-body parton correlation of the hadron structure. In the simplified hypothesis of neglecting all correlations in fractional momenta, the inclusive double parton scattering cross section for the two parton processes $A$ and $B$ reduces nevertheless to a simplest factorized expression:

$$
\sigma_{D}=m \frac{\sigma_{A} \sigma_{B}}{2 \sigma_{e f f}}
$$

where $m=1$ when $A$ and $B$ are indistinguishable processes and $m=2$ when they are distinguishable. $\sigma_{A}$ and $\sigma_{B}$ are the inclusive single scattering cross sections for producing the processes $A$ and $B$ respectively and all the new information on the structure of the

hadron in transverse space is summarized in the value of the scale factor $\sigma_{\text {eff }}$. This simplest hypothesis has not been contradicted by the experimental evidence [2], whose results are in fact described with a single parameter (the scale factor $\sigma_{e f f}$ ). The experimental value quoted by CDF, $\sigma_{\text {eff }}=14.5 \pm 1.7_{-2.3}^{+1.7} \mathrm{mb}$, is however too small to be understood in a simplest uncorrelated picture of the multi-parton distribution and indicates that correlations in transverse space play an important role [3]. A possibility which has been considered [4] to explain the smallness of the value of $\sigma_{\text {eff }}$ is to correlate the population of gluons and sea quarks with the configuration of the valence in transverse space, in such a way that the average number of gluons and sea quarks is small when the valence quarks are all close in 
transverse space and, on the contrary, is large when they are separated by a (relatively) large transverse distance. Such a mechanism increases the dispersion in the number of multiple parton collisions and, as a consequence, $\sigma_{D}$ (which is proportional to that dispersion 《4]). The value of $\sigma_{e f f}$ is therefore diminished with respect to the uncorrelated case. The scale factor, on the other hand, is the result of the overlap in transverse space of the matter distribution of the two interacting hadrons and a feature of the model above is that the average transverse distance of a pair of valence quarks is different as compared with the average transverse distance of a pair of gluons or sea quarks. In the model $\sigma_{e f f}$ is therefore different for double parton scatterings involving valence quarks and for double parton scattering involving sea quarks and gluons. Although the details of the model should be understood in a qualitative rather than in a quantitative sense, it is rather natural to expect different values of the scale factor in different reactions. We think therefore that it may be interesting to have an indication, even if only qualitative, on the size of the effect and, to that purpose, we work out in the present note the values of the scale factor foreseen in the model [4] in a few cases of interest.

\section{SCALE FACTOR IN A CORRELATED MODEL}

At large energies one will be able to observe double parton collisions in various channels [5] [6] [7]. In particular the production of two equal sign $W$ bosons, at relatively low $p_{t}$, goes almost entirely through double parton collisions [7] and is originated, to a large extent, by interactions with valence quarks. Another case where double parton collisions are expected to play an important role is in the production of two $b \bar{b}$ pairs. The corresponding integrated cross section is in fact rather large, by using Eq.1] one may estimate that the double parton scattering cross section for producing two $b \bar{b}$ pairs is of the order of $10 \mu \mathrm{b}$ at the energy of the LHC. The dominant mechanism of $b \bar{b}$ production is gluon fusion and the observation of double parton collisions, in a equal sign $W$ boson pair production and in the production of two $b \bar{b}$ pairs, allows therefore one to compare the distribution in transverse space of valence 
quarks with the distribution in transverse space of gluons.

To work out the scale factor in the two cases we write the inclusive double parton scattering cross section, for the parton interactions $A$ and $B$, as

$$
\sigma_{D}(A, B)=\frac{m}{2} \sum_{i j k l} \int_{p_{t}^{c u t}} \Gamma_{i j}\left(x_{1}, x_{2} ; b\right) \hat{\sigma}_{i k}^{A}\left(x_{1}, x_{1}^{\prime}\right) \hat{\sigma}_{j l}^{B}\left(x_{2}, x_{2}^{\prime}\right) \Gamma_{k l}^{\prime}\left(x_{1}^{\prime}, x_{2}^{\prime} ; b\right) d x_{1} d x_{1}^{\prime} d x_{2} d x_{2}^{\prime} d^{2} b
$$

where $\Gamma_{i j}\left(x_{1}, x_{2} ; b\right)$ are the double parton distributions, depending on the two fractional momenta of the partons $x_{1}$ and $x_{2}$ and on their relative transverse distance $b$. The indices $i$ and $j$ refer to the different kinds of partons, $\hat{\sigma}_{i k}^{A}$ and $\hat{\sigma}_{j l}^{B}$ are the partonic cross sections and the QCD dependence on the scale of the interaction is implicit in all quantities. In the case we are considering the dependence of $\Gamma$ on $x_{1}, x_{2}$ and $b$ is factorized:

$$
\Gamma_{i j}\left(x_{1}, x_{2} ; b\right)=G_{i}\left(x_{1}\right) G_{j}\left(x_{2}\right) F_{j}^{i}(b)
$$

where $G_{i}(x)$ are the usual parton distributions. If $F_{j}^{i}(b)$ do not depend on $i$ and $j$ one obtains Eq.1] and the scale factor $\sigma_{e f f}$ is universal. In general the double scattering cross section is however written as

$$
\sigma_{D}(A, B)=\frac{m}{2} \sum_{i j k l} \Theta_{k l}^{i j} \sigma_{i j}(A) \sigma_{k l}(B)
$$

where $\sigma_{i j}(A)$ is the hadronic inclusive cross section for two partons of kind $i$ and $j$ to undergo the hard interaction $A$, and

$$
\Theta_{k l}^{i j}=\int d^{2} b F_{k}^{i}(b) F_{l}^{j^{\prime}}(b)
$$

are geometrical coefficients, with dimensions of the inverse of a cross section, depending on the various parton processes.

In the model [4] the probability to find the proton in a given configuration, with $n$ gluons or sea-quarks, has the following expression:

$$
\begin{aligned}
& P\left(X_{v},\right.\left.\mathbf{B}_{v} ; x_{1}, \mathbf{b}_{1}, \ldots x_{n}, \mathbf{b}_{n}\right)=q_{v}\left(X_{1}\right) q_{v}\left(X_{2}\right) q_{v}\left(X_{3}\right) \varphi\left(\mathbf{B}_{D}, \mathbf{B}\right) \\
& \times \frac{1}{n !}\left[g\left(x_{1}\right) f\left(B_{D}, b_{1}\right) \ldots g\left(x_{n}\right) f\left(B_{D}, b_{n}\right)\right] \exp \left\{-\frac{B_{D}^{2}}{\left\langle B_{D}^{2}\right\rangle} \int g(x) d x\right\}
\end{aligned}
$$


where $q_{v}(X)$ are the inclusive distributions of valence quarks, as a function of the momentum fraction $X$, and $\varphi\left(\mathbf{B}_{D}, \mathbf{B}\right)$ is the density of valence quarks in transverse space, where the coordinates of valence quarks in transverse space $\mathbf{B}_{v}$ are given by

$$
\begin{aligned}
\mathbf{B}_{1} & =\frac{1}{2} \mathbf{B}_{D}+\mathbf{B} \\
\mathbf{B}_{2} & =\frac{1}{2} \mathbf{B}_{D}-\mathbf{B} \\
\mathbf{B}_{3} & =-\mathbf{B}_{D}
\end{aligned}
$$

In the model $\varphi\left(\mathbf{B}_{D}, \mathbf{B}\right)=\int d Z_{D} d Z \phi\left(\mathbf{R}_{D}, \mathbf{R}\right)$, where $\phi\left(\mathbf{R}_{D}, \mathbf{R}\right)$ represents the distribution density of the proton in coordinate space. The explicit expression used is

$$
\phi\left(\mathbf{R}_{D}, \mathbf{R}\right)=\frac{\lambda_{D}^{3} \lambda^{3}}{(8 \pi)^{2}} \exp \left\{-\left(\lambda_{D} R_{D}+\lambda R\right)\right\}
$$

where

$$
\begin{gathered}
\lambda_{D}=\frac{2 \sqrt{3}}{\sqrt{\left\langle r^{2}\right\rangle}} \\
\lambda=\frac{4}{\sqrt{\left\langle r^{2}\right\rangle}}
\end{gathered}
$$

with $\sqrt{\left\langle r^{2}\right\rangle}=0.81 \mathrm{fm}$, the proton charge radius.

Given a configuration of the valence, the average density of gluons and sea quarks in a point with transverse coordinate $b$ and momentum fraction $x$ is given by $g(x) f\left(B_{D}, b\right)$. The overall average number of gluons and sea quarks at a given $x$ (namely after integrating on $b$ and on the configurations of the valence) is $g(x)$ and is identified with the inclusive distribution of gluons and sea quarks. In the same way the inclusive distributions of the valence quarks, $q_{v}(X)$, are the result of integrating over the transverse coordinates $\mathbf{B}_{v}$ and of summing over all configurations of gluons and sea quarks.

The dependence of the average density of gluons and sea quarks on the transverse coordinate $b$ is expressed by

$$
f\left(B_{D}, b\right)=\frac{3}{2 \pi}\left(1-\frac{b^{2}}{B_{D}^{2}}\right)^{1 / 2} \frac{1}{\left\langle B_{D}^{2}\right\rangle} \theta\left(B_{D}-b\right)
$$


which is the projection on the transverse plane of a sphere of radius $B_{D}$, rescaled with the factor $B_{D}^{2} /\left\langle B_{D}^{2}\right\rangle$, where the average $\left\langle B_{D}^{2}\right\rangle$ is taken with the density $\varphi\left(\mathbf{B}_{D}, \mathbf{B}\right)$. The average density of gluons and sea quarks and the corresponding average number (which grows as $\left.B_{D}^{2} /\left\langle B_{D}^{2}\right\rangle\right)$ are therefore correlated with the configuration of the valence, while all fractional momenta are uncorrelated.

The model distinguishes two different kinds of partons, as far as the number and density in transverse space are concerned, the valence quarks and the sea quark and gluons. The indices $i$ and $j$, as a consequence, can take two different values, $v$ and $s$, and the relevant transverse densities of parton pairs to be considered are

$$
\begin{aligned}
& F_{v}^{v}(b)=\frac{1}{6} \sum_{j \neq i=1}^{3} \int d^{2} B d^{2} B_{D} \varphi\left(\mathbf{B}_{D}, \mathbf{B}\right) \delta\left(\mathbf{B}_{i}-\mathbf{B}_{j}-\mathbf{b}\right) \\
& F_{s}^{v}(b)=\frac{1}{3} \sum_{i=1}^{3} \int d^{2} B d^{2} B_{D} d^{2} b^{\prime} \varphi\left(\mathbf{B}_{D}, \mathbf{B}\right) f\left(B_{D}, b^{\prime}\right) \delta\left(\mathbf{B}_{i}-\mathbf{b}^{\prime}-\mathbf{b}\right) \\
& F_{s}^{s}(b)=\int d^{2} B d^{2} B_{D} d^{2} b^{\prime} d^{2} b^{\prime \prime} \varphi\left(\mathbf{B}_{D}, \mathbf{B}\right) f\left(B_{D}, b^{\prime}\right) f\left(B_{D}, b^{\prime \prime}\right) \delta\left(\mathbf{b}^{\prime}-\mathbf{b}^{\prime \prime}-\mathbf{b}\right)
\end{aligned}
$$

By using Eq.5 and the expression below, the matrix $\Theta_{k l}^{i j}$ and the effective cross section are readily evaluated:

$$
\sum_{i j k l} \Theta_{k l}^{i j} \sigma_{i j}(A) \sigma_{k l}(B)=\frac{\sigma(A) \sigma(B)}{\sigma_{e f f}}
$$

\section{RESULTS}

The resulting values of $1 / \Theta_{k l}^{i j}$ are shown in the table. The scale factor $\sigma_{e f f}$ is plotted as a function of the c.m. energy in fig. 1 and in fig.2, corresponding to $p p$ and $p \bar{p}$ interactions respectively, in various processes of interest: $i$ ) production of two equal sign $W$ bosons, ii) production of a $W$ boson of either positive or negative sign together with two jets or iii) with a $b \bar{b}$ pair, $i v$ ) production of four jets, $v$ ) production of two $b \bar{b}$ pairs. In the case of jets the scale factor has been evaluated by using as a lower cutoff in $p_{t}$ the value of $5 \mathrm{GeV}$ (the scale factor is however rather insensitive to that choice). All the single scattering cross sections in Eq.12 have been computed at the lowest order in the coupling constant 
and by making use of the Martin-Roberts-Stirling 1999 (MRS99) parton distributions [8]. The different results obtained for $W^{+} W^{+}$and $W^{-} W^{-}$production for $p p$ collisions is mainly due to the different content of $d$ and $u$ quarks in the proton. A consequence is in fact the different contribution of the sea quarks in the two cases, whose distribution is sizably different in the model in comparison with the distribution in transverse space of the valence. To check the dependence on the choice of the distribution functions we have repeated the calculation by using the CTEQ5 parton distributions [9]. In all cases the scale factor is not

changed by more than a few percent. In fact the results for $\sigma_{\text {eff }}$ are rather insensitive to the choice of parton distributions, since $\sigma_{e f f}$ is obtained by making ration of cross sections. The main qualitative feature of the results obtained is the strong difference, at Tevatron energy, between final states with and without a $W$ boson, and the energy dependence of the scale factor, which is sizable when moving from Tevatron to LHC energy in the channels containing a $W$ boson.

While the actual values should be considered only as indicative, the qualitative features just pointed out are likely to be of more general validity, since they are originated by the different source of initial state partons in the case of $W$ production, which in most cases involves valence quarks, in comparison with the other channels considered, that are mainly generated by interactions of sea quarks and gluons. The overall indication is that it is plausible to expect non minor differences in the values of $\sigma_{e f f}$ in different processes. It is also apparent that a basic element, to understand the three dimensional structure of the proton, is a quantitative information on the correlations in transverse space of the various pairs of initial state partons and that the scale factors, of the different double parton collision processes, are the physical observables which can provide that information.

\section{Acknowledgment}

This work was partially supported by the Italian Ministry of University and of Scientific and Technological Researches (MURST) by the Grant COFIN99. 


\section{TABLES}

TABLE I. Scale factors $1 / \Theta_{k l}^{i j}(\mathrm{mb})$

\begin{tabular}{lc}
\hline \hline Partons $(\mathrm{ik}-\mathrm{jl})$ & Scale factor \\
\hline (ss-ss) & 12.4 \\
$(\mathrm{vs}-\mathrm{ss})$ & 31.9 \\
$(\mathrm{vv}-\mathrm{ss})$ & 28.3 \\
$(\mathrm{vs}-\mathrm{vs})$ & 69.4 \\
$(\mathrm{vs}-\mathrm{sv})$ & 69.4 \\
$(\mathrm{vv}-\mathrm{vs})$ & 68.3 \\
$(\mathrm{vv}-\mathrm{vv})$ & 67.4 \\
\hline \hline
\end{tabular}




\section{FIGURES}

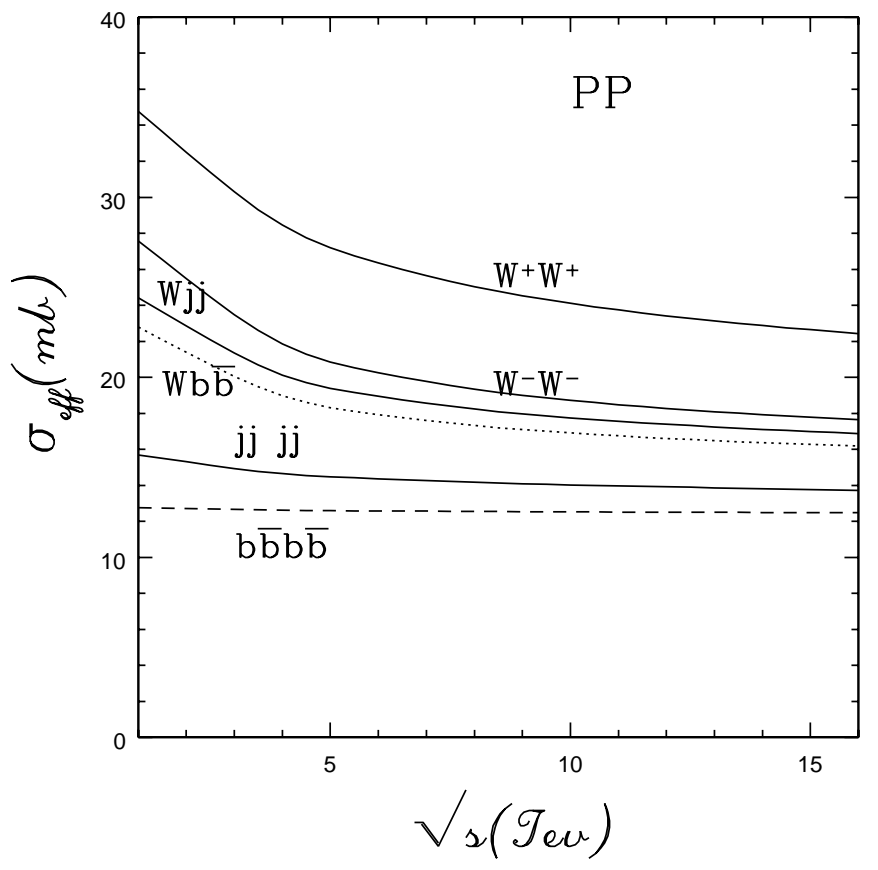

FIG. 1. $\sigma_{e f f}$ as a function of the c.m. energy in various processes in $p p$ collisions. 


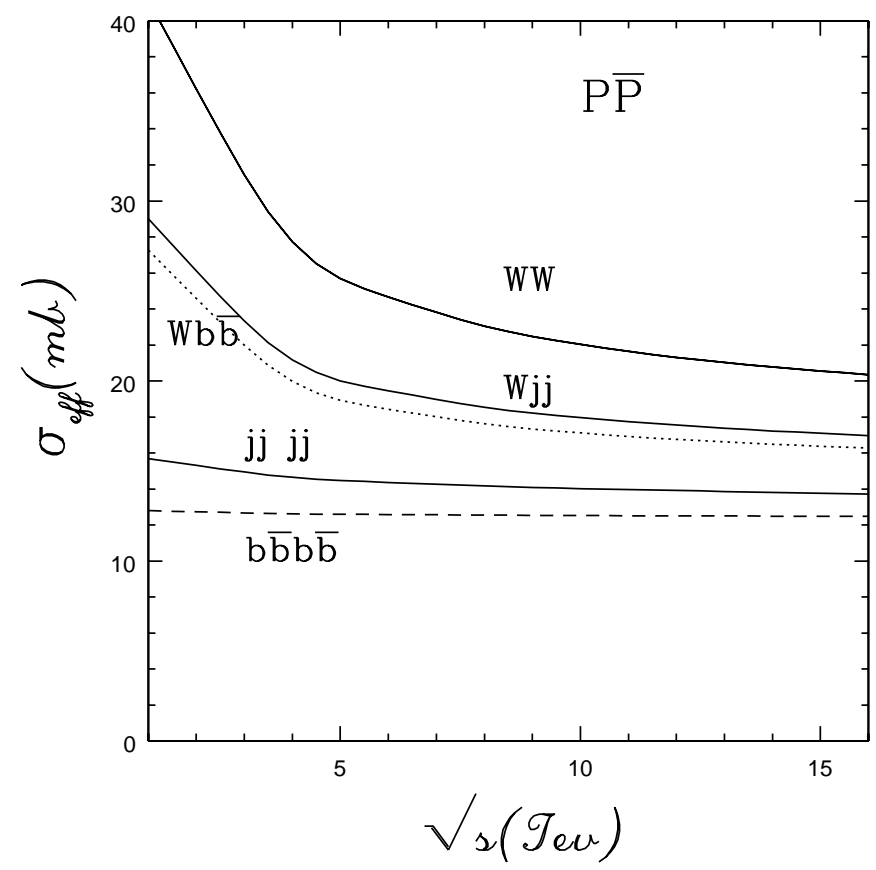

FIG. 2. $\sigma_{\text {eff }}$ as a function of the c.m. energy in various processes in $p \bar{p}$ collisions. 


\section{REFERENCES}

[1] P.V. Landshoff and J.C. Polkinghorne,Phys. Rev. D18 3344 (1978); Fujio Takagi Phys. Rev. Lett. 43, 1296 (1979); C. Goebel, F. Halzen and D.M. Scott, Phys. Rev. D22, 2789 (1980); N. Paver and D. Treleani, Nuovo Cimento A70, 215 (1982); B. Humpert, Phys. Lett. B131, 461 (1983); M. Mekhfi, Phys. Rev. D32, 2371 (1985), ibid. D32, 2380 (1985); B. Humpert and R. Odorico, Phys. Lett. 154B, 211 (1985); T. Sjostrand and M. Van Zijl, Phys. Rev. D36, 2019 (1987); F. Halzen, P. Hoyer and W.J. Stirling Phys.Lett. 188B, 375 (1987); M. Mangano, Z. Phys. C42, 331 (1989); R.M. Godbole, Sourendu Gupta and J. Lindfors, Z. Phys. C47 69 (1990).

[2] F. Abe et al. (CDF Collaboration), Phys.Rev.Lett. 79584 (1997); Phys. Rev. D56 3811 (1997).

[3] G. Calucci and D. Treleani, Phys. Rev. D57 503 (1998).

[4] G. Calucci and D. Treleani, Phys. Rev. D60 (1999) 054023.

[5] S. Catani, M. Dittmar, D. Soper, W.J. Stirling, S. Tapprogge, S. Alekhin, P. Aurenche, C. Balazs, R.D. Ball, G. Battistoni, E.L. Berger, T. Binoth, R. Brock, D. Casey, G. Corcella, V. Del Duca, A. Del Fabbro, A. De Roeck, C. Ewerz, D. de Florian, M. Fontannaz, S. Frixione, W.T. Giele, M. Grazzini, J.P. Guillet, G. Heinrich, J. Huston, J. Kalk, A.L. Kataev, K. Kato, S. Keller, M. Klasen, D.A. Kosower, A. Kulesza, Z. Kunszt, A. Kupco, V.A. Ilyin, L. Magnea, M.L. Mangano, A.D. Martin, K. Mazumdar, Ph. Mine, M. Moretti, W.L. van Neerven, G. Parente, D. Perret-Gallix, E. Pilon, A.E. Pukhov, I. Puljak, J. Pumplin, E. Richter-Was, R.G. Roberts, G.P. Salam, M.H. Seymour, N. Skachkov, A.V. Sidorov, H. Stenzel, D. Stump, R.S. Thorne, D. Treleani, W.K. Tung, A. Vogt, B.R. Webber, M. Werlen, S. Zmouchko. CERN-TH-2000-131, hep-ph/0005025.

[6] A. Del Fabbro and D. Treleani, Phys. Rev. D61 (2000) 077502.

[7] A. Kulesza and W.James Stirling, Phys. Lett. B475 (2000) 168. 
[8] A.D.Martin, R.G.Roberts, W.J.Stirling and R.S.Thorne, Eur. Phys. J. C14 (2000) 133.

[9] H. L. Lai, J. Huston, S. Kuhlmann, J. Morfin, F. Olness, J. F. Owens, J. Pumplin, W. K. Tung, Eur. Phys. J. C12 (2000) 375. 